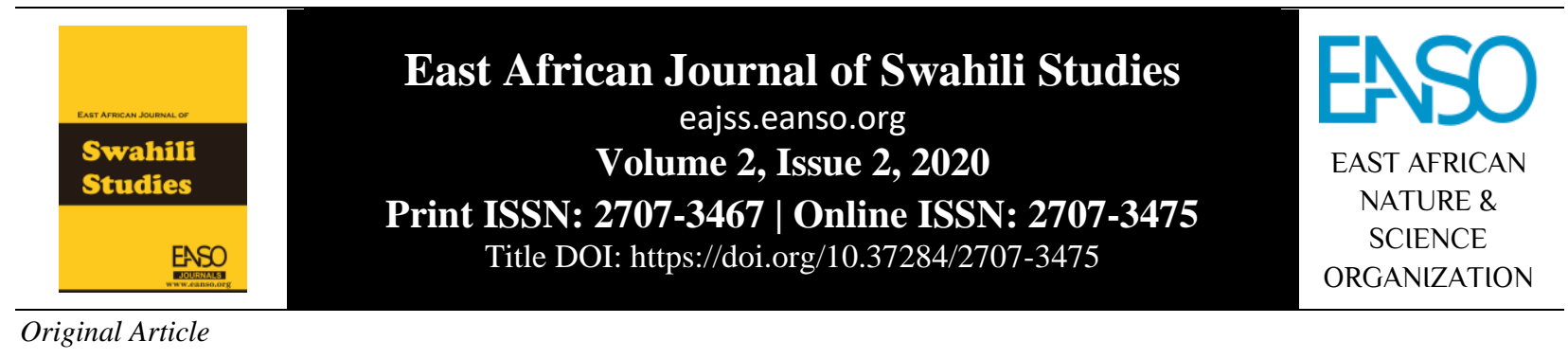

\title{
Maudhui katika Nyimbo Teule Kwenye Kipindi cha Rauka
}

\author{
Joyce Chepkwony ${ }^{*}$, Dkt. Fred Wanjala Simiyu ${ }^{2}$ \& Margan Adero ${ }^{1}$ \\ ${ }^{1}$ Catholic University of Eastern Africa, SLP 62157 - 00200, Nairobi, Kenya. \\ ${ }^{2}$ Kibabii University, SLP 1699 - 50200, Bungoma, Kenya. \\ *Barua pepe ya mawasiliano: joycemaiyo555@gmail.com
}

DOI ya Nakala: https://doi.org/10.37284/eajss.2.2.183

Tarehe ya Uchapishaji: IKISIRI

15 July 2020 Utafiti huu unahusu maudhui katika nyimbo teule kwenye vyombo vya habari, mfano ni nyimbo katika kipindi cha Rauka katika runinga ya Citizen. Utafiti

Maneno Muhimu:

Maudhui,

Nyimbo,

Kipindi,

Rauka. ulichochewa na haja ya kutaka kuchunguza nafasi ya vyombo vya habari katika kuhuisha fasihi simulizi kupitia utanzu wa nyimbo. Tafiti za awali zilichunguza tanzu nyingine kama mazungumzo na maigizo. Utafiti huu ulidhamiria hususan: Kubainisha vigezo vilivyotumiwa kuteua nyimbo zinazochezwa kwenye kipindi cha Rauka; Kuonyesha maudhui katika nyimbo teule; Kuchunguza mbinu za lugha zilizotumiwa kuwasilisha maudhui za nyimbo zinazoteuliwa na kuchezwa kwenye kipindi cha Rauka. Utafiti huu ulifanywa kwa kuzingatia muundo wa kimfano unaoeleza kuwa mtafiti huteua eneo au kitu maalum ambacho kitashughulikiwa na kuwezesha kupatikana kwa data za utafiti zilizokusudiwa. Utafiti huu uliongozwa na nadharia ya Ajenda iliyoasisiwa na McCombs na Shaw mwaka wa 1972. Nadharia ya Ajenda ilitumika kuhakiki uteuzi wa nyimbo wa kipindi kinachopeperushwa na runinga. Uchunguzi ulifanywa katika idhaa ya Citizen kipindi cha Rauka kinachopeperusha nyimbo za dini. Utafiti uliwahusisha waandaaji wawili wa kipindi cha Rauka cha runinga ya Citizen na watazamaji 68 kutoka eneo la Eldoret, Kaunti ya Uasin Gishu, shule za upili ya wasichana ya Hill School. Kwa ujumla, utafiti uliwahusisha watu 70. Watazamaji waliteuliwa kwa kutumia usampulishaji kimaksudi. Vifaa vifuatavyo vilitumiwa kukusanya data: hojaji kwa watazamaji, usaili kwa waandaaji wa kipindi na mwalimu msimamizi wa masuala ya dini na mkuu wa ushauri na nasaha na kinasa sauti kunasia vipindi vilivyoteuliwa kisha vikachanganuliwa. Data ifuatayo ilikusanywa: Nyimbo zilizochezwa kwenye kipindi cha Rauka ziliteuliwa kwa kuzingatia mada ya siku, muda uliotengewa kipindi cha Rauka, hadhira, masuala ibuka, kuwepo na nyimbo zinazowavutia watazamaji; Utafiti 
umebainisha kuwa nyimbo zinazochezwa katika kipindi cha Rauka zina maudhui ya upendo, shukrani, msamaha, uvumilivu, heshima, wema na msaada. Data iliyokusanywa ilichanganuliwa kwa kuzingatia nadharia ya Ajenda kwa kubainisha maudhui yaliyomo kisha matokeo yakawasilishwa kupitia maelezo ya kifafanuzi na mfano. Matokeo ya utafiti huu yatakuwa mchango kwa tafiti ambazo zimefanywa kuhusu fasihi simulizi na matumizi yake katika vyombo vya habari. Utafiti huu vilevile utakuwa wa manufaa kwa yeyote atakayefanya utafiti kuhusu fasihi simulizi na vyombo vya habari.

\section{APA CITATION}

Chepkwony, J., Simiyu, F., \& Adero, M. (2020). Maudhui katika Nyimbo Teule Kwenye Kipindi cha Rauka. East African Journal of Swahili Studies, 2(2), 32-43. https://doi.org/10.37284/eajss.2.2.183

\section{CHICAGO CITATION}

Chepkwony, Joyce, Fred Simiyu, and Margan Adero. 2020. "Maudhui katika Nyimbo Teule Kwenye Kipindi cha Rauka". East African Journal of Swahili Studies 2 (2), 32-43. https://doi.org/10.37284/eajss.2.2.183.

\section{HARVARD CITATION}

Chepkwony, J., Simiyu, F. and Adero, M. (2020) "Maudhui katika Nyimbo Teule Kwenye Kipindi cha Rauka", East African Journal of Swahili Studies, 2(2), pp. 32-43. doi: 10.37284/eajss.2.2.183.

\section{IEEE CITATION}

J. Chepkwony, F. Simiyu, and M. Adero, "Maudhui katika Nyimbo Teule Kwenye Kipindi cha Rauka", EAJSS, vol. 2, no. 2, pp. 32-43, Jul. 2020.

\section{MLA CITATION}

Chepkwony, Joyce, Fred Simiyu, and Margan Adero. "Maudhui katika Nyimbo Teule Kwenye Kipindi cha Rauka". East African Journal of Swahili Studies, Vol. 2, no. 2, July 2020, pp. 32-43, doi:10.37284/eajss.2.2.183.

\section{UTANGULIZI}

Utafiti huu unachunguza maudhui katika nyimbo teule zinazochezwa kwenye kipindi cha Rauka kinachopeperushwa kila Jumapili kuanzia saa kumi na mbili hadi saa tatu asubuhi kwenye runinga ya Citizen. Fasihi ni kioo cha jamii na huwasilisha ujumbe kupitia tanzu mbalimbali za fasihi.

Senkoro, (1979) anaeleza maana ya maudhui kuwa ni jumla ya mawazo yote yanayozungumzwa pamoja na mitazamo ya mwandishi juu ya mawazo hayo. Maudhui hujumuisha mawazo pamoja na mafunzo mbalimbali yaliyomsukuma msanii hadi akatunga na kusanii kazi fulani ya kifasihi. Vipengele vya maudhui ni pamoja na dhamira, migogoro, falsafa, ujumbe na msimamo. Fasihi huwasilisha maudhui kupitia nyimbo kama mojawapo za tanzu za fasihi na hutekeleza majukumu mbalimbali katika jamii kwani ni rahisi sana kuteka makini ya hadhira.

Kulingana na Wamitila (2008) maudhui ni jumla ya masuala au mambo yanayoshughulikiwa katika kazi ya kifasihi kama vile nyimbo. Maudhui hujumuisha dhamira, itikadi, msimamo, maadili, mafunzo na falsafa.

Wataalamu mbalimbali wametoa maelezo kuhusu maana ya nyimbo. Kwa mujibu wa Beja (2001) wimbo ni utungo wa fasihi simulizi unaowasilisha ujumbe kwa kutumia sauti na matendo ya waimbaji.

Matteru (1981) anasema kuwa nyimbo ni maneno yanayotamkwa kwa sauti ya kuvutia na kwa mahadhi ya kupendeza. Anaendelea kusema kuwa nyimbo ni aina ya shairi zinazoimbwa pekee au kuhusishwa na ngoma. Inabainika wazi kuwa nyimbo zimekuwemo miongoni mwa binadamu hata kabla ya maandishi kuvumbuliwa na imekuwa ni mojawapo wa njia za watu kujitumbuiza katika sherehe mbalimbali. Nyimbo vilevile hutumiwa kupitisha ujumbe kwa watu wenye umri tofauti katika jamii.

Nyimbo ni aina ya tanzu za fasihi simulizi. Fasihi simulizi ni sanaa ambayo hutumia lugha hasa maneno ambayo yametamkwa kupitia kinywa cha 
binadamu. Nyimbo ni mali za jamii. Rekodi za matukio muhimu katika jamii huhifadhiwa kwa nyimbo na kupokezwa kwa vizazi. Nyimbo hutumiwa kupitisha amali na mambo ambayo jamii inayothamini. Amali hizo hurekodiwa katika nyimbo mbalimbali kama vile za kazi na kurithishwa vizazi vya jamii hiyo. Mawaidha na thamani za jamii hukaririwa katika nyimbo.

Wamitila (2003) anaeleza kuwa nyimbo hutokana na mazingira. Mazingira ndiyo yanayozalisha hisia na hatimaye kwa msanii. Hivyo basi, katika nyimbo panapaswa kuwa na wazo maalum ambalo linawasilishwa kwa hadhira husika kwa njia ya sauti. Nyimbo ni utanzu wa kishairi zinazoimbwa kwa muwala fulani. Umaarufu wa muziki katika wimbo huruhusu maana ya maneno kuchukuliwa sawa na uimbaji wa muziki. Nyimbo ni zao la shughuli za binadamu na hisia zake na hivyo huzingatia muktadha mbalimbali. Nyimbo zimefunganishwa na muktadha fulani pale ambapo pana hadhira na matini. Hadhira huwa ni wale wanaosikiliza au kutazama nyimbo nayo matini yakiwa ni yale matamshi yenyewe kwa njia ya uimbaji.

Nyimbo ni aina ya tanzu ya fasihi simulizi. Fasihi simulizi ni sanaa inayotumia lugha, hasa maneno ambayo yametamkwa kupitia kinywa cha binadamu. Nyimbo ni mali za jamii mbalimbali zenye manufaa mengi kwa binadamu na kupitia uwezo wake wa kutamka maneno huwasilisha ujumbe kwa njia ya mazungumzo, kughani na kuimba.

Nyimbo zinaweza kuainishwa katika makundi tofauti kwa kuzingatia vigezo mbalimbali. Kuna nyimbo za watoto, za kazi au hodiya, za mapenzi, za dini, za sifa, za mbolezi na za jando au nyiso. Utafiti huu unashughulikia nyimbo za dini zinazopeperushwa kwenye kipindi cha Rauka. Nyimbo za dini ni zile zinazoimbwa ili kumsifu Mungu kwa ajili ya uwezo alionao katika maisha ya binadamu. Wanjala (2013) anasema kuwa nyimbo hizi hutumiwa katika sehemu za maabadini (kanisani au msikitini) kwa malengo ya kumtukuza Maulana. Kristo, mitume, malaika au mama Maria. Nyimbo hizi hunuia kuwaelekeza watu katika jamii kwa kuwaonya au kuwatia moyo baada ya matatizo fulani kutokea. Nyimbo hizi vilevile huimbwa katika muktadha tofauti kama vile matanga na harusi. Huimbwa kwa unyenyekevu. Sifa za nyimbo za dini ni kama vile: Huimbwa na watu walio na imani fulani kwa Mungu wao na huimbwa pahali maalum kama vile kanisani au msikitini. Aghalabu huandamana na ala mbalimbali za muziki kama vile kengele, ngoma na vinanda. Nyimbo hizi hutaja sifa za Mungu, matendo yake na uhusiano wake na watu. Nyimbo nyingine huwa za ombi au toba na nyingine za kumtukuza Mungu. Huwa na miondoko inayodhihirisha hisia za waumini ambazo huambatana na nyimbo hizi.

Hivyo basi, nyimbo huweza kueleza hisia za binadamu kama vile furaha, kero, matarajio, uzito wa fikira, imani, mila na hali tofauti maishani. Nyimbo zapaswa kuwa na mpangilio maalum wa ujumbe unaowasilishwa kwa hadhira lengwa.

Nchini Kenya, vyombo vya habari vimeipa fasihi nafasi kubwa sana na hivyo basi kupanua hadhira yake. Hii ni kutokana na sababu kuwa fasihi hii inathaminiwa sana na wengi na kupewa nafasi kwenye vyombo vya habari hasa kwa muda mrefu kwenye redio na runinga.

Utafiti tulioufanya ulilenga kuchunguza maudhui katika nyimbo teule kwenye kipindi cha Rauka. Nyimbo ni utanzu wa ushairi unaopatikana katika fasihi simulizi na ambao huchukua nafasi kuu katika jamii. Utanzu huu umepewa fasili nyingi ikiwemo:

Nyimbo ni tungo zenye mahadhi ya sauti inayopanda na kushuka ambapo nyimbo hizi huundwa kwa lugha ya mvuto, matumizi ya taswira na mapigo ya silabi. (Wamitila, 2003).

Katika utafiti wetu, tulichukulia kuwa nyimbo ni zaidi ya sauti ambazo huimbwa na kuchezwa, kwani nyimbo huwasilisha mambo mbalimbali yenye umuhimu katika jamii husika. Sanaa ya nyimbo huonyesha uwezo wa binadamu wa kusimulia au kupasha tajriba yake na ya jamii ya kila siku na kujaribu kuleta maana katika maisha.

Umaarufu wa wimbo fulani hautegemei namna watu wanaufurahia bali uwezo wa kupitisha ujumbe unaowawezesha hadhira kubadilisha mitazamo kuhusu maisha. MacFarland (1990), anaeleza umaarufu wa wimbo kuwa una uwezo wa kubadilisha mielekeo ya watu. Kutokana na mawazo haya, utafiti huu uliona haja ya kubainisha 
ujumbe wa nyimbo zinazochezwa katika kipindi cha Rauka kwenye kituo cha runinga ya Citizen iwapo itabadilisha tabia hasi katika jamii.

Nchini Kenya, vyombo vya habari, vikiwemo redio na televisheni vimeipa fasihi hii nafasi kubwa hivyo basi kupanua hadhira yake. Nyimbo nyingi zinazosikika kwenye vyombo vya habari huwahimiza watu kuwa na uwajibikaji, uzazi wa mpango, kuchagua viongozi bora na kufanya kazi kwa bidii. Jambo hili huufanya utanzu huu kuufikia hadhira pana. Aidha, nyimbo huchukua sehemu kuu katika sherehe na mihadhara mbalimbali, iwe rasmi au isiyo rasmi, hivyo kuifanya fasihi hii kuwa muhimu katika jamii.

Vituo vya runinga visivyo na misingi ya kidini vinatenga muda wa kupeperusha nyimbo za kidini za wasanii mbalimbali. Idhaa ya Citizen haijaambatanishwa na dini maalum. Hata hivyo huwa inatenga muda wa kupeperusha vipindi vya dini, haswa, siku ya Jumapili. Inateua na kucheza nyimbo za kidini hasa kupitia kipindi cha Rauka. Kando na upekee huo wa kupeperusha nyimbo za dini, suala ambalo linahitaji uchunguzi ni namna ambavyo nyimbo hizo huteuliwa na ujumbe unaowasilishwa na nyimbo hizo hasa ikizingatiwa kuwa umaarufu wa muziki ni zaidi ya kufurahia wimbo tu bali umaarufu wake huwa na nguvu ya kubadilisha mielekeo ya watazamaji wake kadri zinavyochezwa kwenye runinga.

Waasisi wa nadharia ya ajenda McCombs na Shaw (1972), walihusisha nadharia hii na tafiti za uchaguzi hasa kupitia taarifa za habari. Tangu kuasisiwa kwake, watafiti wengi walijibwaga nyanjani kuchunguza ukweli wa madai ya nadharia kuwa vyombo vya habari vinaweza kuteua habari zinazochukuliwa kuwa muhimu kwao na kuzipeperusha kila mara ili umma uzichukulie pia kuwa ni muhimu. Waandaaji wa taarifa za habari hufanya hivyo kupitia kwa kusimulia kisa kimoja katika vituo vingi, kuongeza ukubwa wa picha inayohusu habari fulani na kuboresha utangazaji wa kisa husika (Behr \& Lyengar, 1988; Wanta, 1998)

Hata hivyo, nadharia ya ajenda inaweza kutumiwa kwenye miktadha yoyote ambapo watazamaji wanaona umuhimu wa masuala fulani kama vile habari, matangazo ya kibiashara na umaarufu wa nyimbo. MacFarland (1990), anahoji kuwa umaarufu wa wimbo fulani hautegemei tu kufurahia wimbo bali hutegemea pia namna mtazamaji au msikilizaji wa wimbo fulani hubadili mielekeo yake.

Uchunguzi wa suala la ajenda barani Afrika unaonyesha kuwa vyombo vya habari huteua mada na vipindi vya kupeperushwa. Kutokana na uteuzi huo, vyombo vya habari huipa uzito mada maalum na kuwafanya wasikilizaji wawe makini zaidi kuhusu mada hiyo. Uteuzi huo wa mada unaofanywa na vyombo vya habari huwafanya wasikilizaji wazungumzie na hata kuchangia huku wakipuuza mada zingine zilizopo (Wasike, 2017).

Kutokana na uwezo wa wimbo kuibadilisha mielekeo ya watazamaji ni bora kutambua ujumbe unaowasilishwa na nyimbo ili kubaini iwapo mielekeo ya watazamaji itakuwa chanya au hasi kwa sababu hiyo utafiti huu ulitathmini ujumbe unaowasilishwa na nyimbo zinazopeperushwa kwenye kipindi cha Rauka katika runinga ya Citizen ili kuweka wazi ufaafu wa kipindi hicho. Kwa kufanya hivyo tutakuwa tumepanua matumizi ya nadharia ya Ajenda kutoka mawanda ya taarifa za habari na masuala ya kisiasa.

\section{Msingi wa Kinadharia}

Nadharia ya ajenda hueleza uwezo wa vyombo vya habari kuathiri umuhimu wa mada ya umma. Nadharia hii hueleza kuwa habari inayopeperushwa mara kwa mara kuwa ni muhimu. Waasisi wa nadharia hii ni McCombs na Shaw (1972). Nadharia ya Ajenda inahoji kwamba vyombo vya habari huandaa ajenda kwa maoni ya umma kwa kusisitiza mada fulani.

McCombs \& Shaw (1972) walitafiti kampeni za urais mwaka wa 1968, 1972, na 1976. Utafiti wa 1968 waliegemea mambo mawili; Kuwajuza na kuhamasisha. Kwa kutumia nadharia hii walichunguza uhusiano kati ya kilichosemwa na wapiga kura katika jamii na kilichomo kwenye vyombo vya habari wakati wa kampeni. Walihitimisha kuwa vyombo vya habari vilikuwa na jukumu kubwa la kuathiri maoni ya umma kuhusu masuala muhimu.

Lippman alieleza kuwa vyombo vya habari huleta mahusiano ya matukio na picha kwenye akili za umma. Katika maelezo yake, alizungumzia nadharia ya ajenda bila kujua. Mwaka wa 1993, 
Benard Cohen alieleza kuwa vyombo vya habari huathiri maoni ya umma kuhusu jambo linalofaa kuchukuliwa kuwa muhimu. Mwaka 1960, Cohen alikuwa wa kwanza kuelezea namna vyombo vya habari huathiri maamuzi ya umma kuhusu kinachofikiriwa kuwa muhimu na baadaye kuhidhinishwa na McCombs na Shaw (1972) kama nadharia.

Maxwell McCombs alipitia kazi ya Cohen na kazi hii ikamwathiri wakiwa na Donald Shaw. Ilizinduliwa rasmi na McCombs na Shaw wakati wa uchaguzi wa urais kule Chapel Hill, North Carolina mwaka wa 1968 McCombs na Shaw ni wa kwanza kuthibitisha kuwa vyombo vya habari vinaweza kuathiri uamuzi wa umma katika kutoa maoni kuhusu masuala ya uchaguzi.

Fankhouser alifanya utafiti unaokaribiana naye McCombs na Shaw. Watafiti hawa watatu waliwasilisha matokeo kwenye kongamano la wasomi. Makala ya Fankhouser yalichapishwa baada ya McCombs \& Shaw kuchapishwa. Kulingana na Everett Rogers, makala ya Funkhouser haikuwa bora kwa sababu hakuipa jina nadharia yake na pia hakufanya utafiti wa kutosha. Kulingana na McCombs, habari muhimu hupewa kipaumbele na kupeperushwa kwa muda mrefu wa muda uliotengewa habari hizo na kupewa uzito. Habari hizi zikirudiwa tena na tena huonyesha umuhimu wa mada fulani. Hata hivyo, vyombo vya habari vinaweza tu kutayarisha ajenda iwapo habari zao zitakuwa na umuhimu kwa umma. Nadharia ya Ajenda ilifaa katika utafiti wetu kwa kuwa tuliangazia namna uteuzi wa nyimbo zinazopeperushwa na vyombo vya habari kupitia runinga ya Citizen kipindi cha Rauka hufanywa kwa hiari ya waandaaji wa kipindi ama kwa hiari ya watazamaji. Nadharia hii ina mihimili ifuatayo:

Mhimili wa kwanza hueleza kuwa vyombo vya habari havidhihirishi uhalisia wa habari bali huchuja kwa kuteua zile habari zinazochukuliwa kuwa ni muhimu na kupeperusha. Hapa vyombo vya habari huteua mada ya kupeperusha kwa watazamaji. Kutokana na uteuzi huo, vyombo vya habari huipa uzito mada maalum na kuwafanya watazamaji wawe makini zaidi kuhusu mada hiyo. Kwa mujibu wa mwandaaji wa kipindi aliyehusika kutoa majibu ya maswali ya usaili, alieleza kuwa uteuzi wa mada ya siku unaongozwa na suala ibuka au jambo linalotiliwa maanani kwa siku husika. Kwa mfano, nchini Kenya, kila tarehe 20 mwezi wa kumi wa kila mwaka ni siku ya kusherehekea mashujaa. Kwa hivyo, mada ya siku ilikuwa Ushujaa. Waandaaji wa kipindi wanahusika katika uteuzi wa mada na nyimbo zinazoambatana na mada hiyo. Uteuzi wa mada na nyimbo zinazochezwa kwenye kipindi cha Rauka unaofanywa na vyombo vya habari huwafanya watazamaji wazungumzie na hata kuchangia huku wakipuuza mada zingine. Waandaaji wa kipindi cha Rauka hupeperusha zile nyimbo ambazo zinaambatana na mada ya siku ambazo wenyewe kama waandaaji wa kipindi huchukulia kuwa zina ujumbe muhimu.

Mhimili wa pili hueleza kuwa vyombo vya habari hushughulikia masuala machache. Mhimili huu hueleza kuwa vyombo vya habari huteua masuala na kupeperusha kwa watazamaji ambao wanachukulia kuwa masuala hayo tu ndiyo muhimu. Vile vile, udhibiti wa nyimbo ambazo hupeperushwa na vyombo vya habari unajitokeza. Kila mara wakati wa kipindi, lazima pawepo na mada ya siku hivyo, nyimbo zinazochezwa ni lazima ziambatane na mada iliyoteuliwa. Japo kuna nyimbo nyingi za dini zilizoimbwa na wasanii mbalimbali, waandaaji wa kipindi huteua nyimbo chache na kupeperusha na hivyo watazamaji kuchukulia kuwa nyimbo hizo ni muhimu.

Mhimili wa tatu hueleza kuwa wahariri ndio wanateua habari ya kupeperushwa kwenye vyombo vya habari. Katika mhimili huu, kuwepo kwa wahariri hudhibiti masuala ya kuangaziwa kwenye vyombo vya habari. Udhibiti wa aina za nyimbo ambazo hupeperushwa na vyombo vya habari unajitokeza. Kwa mfano, kuna muda maalum na mada zilizotengewa kipindi, hivyo wahariri huhusika kuteua nyimbo kulingana na muda na pia watazamaji (hadhira). Hapa, vyombo vya habari vinapaswa kuzingatia muda huo ambao umependekezwa kusudi nyimbo zote teule za siku zipeperushwe. Kutokana na hali hiyo panatokea udhibiti wa nyimbo zinazopeperushwa na vyombo vya habari. Katika kipindi cha Rauka, nyimbo zinazopeperushwa wakati wa kipindi huteuliwa na mhariri kabla ya waandaaji wa kipindi kupeperusha. 
Mhimili wa nne ni ajenda ya umma. Katika mhimili huu, umma una mchango wa masuala ya kuangaziwa kwenye vyombo vya habari. Nyimbo nyingi hupeperushwa kwa mfano katika kipindi cha Rauka, umma hupewa nafasi ya kuchagua wimbo bora kati ya kumi ambazo huteuliwa na waandaaji. Hivyo baadhi ya nyimbo zinazochezwa na kupeperushwa kwenye kipindi hupendekezwa na watazamaji kupitia ujumbe mfupi kipindi kinapoendelea. Watazamaji pia huhusika katika uchaguzi wa nyimbo za kuchezwa wakati kipindi kinaendelea.

Utafiti ulitumia mihimili yote minne ya Nadharia ya Ajenda ya McCombs \& Shaw (1972). Hapa, vyombo vya habari huteua nyimbo na kupeperusha kwenye kipindi. Kutokana na uteuzi huo, vyombo vya habari huipa uzito nyimbo maalum na kuwafanya watazamaji wawe makini zaidi kuhusu ujumbe wa nyimbo hizo. Uteuzi wa nyimbo unaofanywa na vyombo vya habari huwafanya watazamaji waimbe na hata kuzitafuta kanda za nyimbo hizo ili wazisikilize mara kwa mara kuliko nyimbo zingine ambazo hazipeperushwi kwenye vyombo vya habari.

Utafiti ulitumia Nadharia ya Ajenda kuchambua maudhui ya nyimbo teule katika kipindi cha Rauka. Matumizi ya Nadharia ya Ajenda yalisaidia katika uteuzi wa nyimbo za kuchanganuliwa. Ingawa watazamaji hutazama nyimbo za kipindi husika, uteuzi wa nyimbo za kupeperushwa huwa mikononi mwa waandaaji na wahariri wa vituo vya runinga. Utafiti huu ulichunguza namna vyombo vya habari vinaweza vikatumiwa kwa njia ya kuifikia umma kupitisha ujumbe wa kukashifu matendo ya uhalifu kupitia uhuishaji wa nyimbo zinazopeperushwa.

\section{MAUDHUI KATIKA NYIMBO TEULE KWENYE KIPINDI CHA RAUKA}

Ilibainika kwamba kupitia kipindi, watazamaji waliweza kupata ujumbe kuhusu upendo, uvumilivu, shukrani, mateso, msaada, heshima na wema. Wengi wa watazamaji wa kipindi cha Rauka waliohusishwa katika utafiti huu walihoji kwamba kipindi hicho kiliwafaa sana kwani kiliwasilisha masuala yaliyoathiri jamii. Kwa mfano, tendo la mateso ambalo linashuhudiwa mara kwa mara katika jamii zetu za akina mama kuumizwa katika ndoa umekashifiwa katika vyombo vya habari kupitia nyimbo zinazopeperushwa. Idhaa ya Citizen kipindi cha Rauka ndiyo iliyoamua mada ya kujadiliwa na nyimbo za kupeperushwa kama inavyojitokeza katika Nadharia ya Ajenda (McCombs \& Shaw) kwamba, vyombo vya habari huteua masuala ya kujadiliwa katika vyombo kama vile magazeti, runinga na redio. Hata hivyo, kupitia nyimbo zinazopeperushwa, ujumbe uliokusudiwa kuwafikia watazamaji uliweza kuangaziwa. Maudhui yaliyojitokeza ni:

\section{Upendo}

Maudhui ya upendo yanajitokeza katika nyimbo teule za dini zinazochezwa kwenye stesheni ya Citizen kipindi cha Rauka. Kwa mujibu wa kamusi ya Sosholojia ya Collins (1979), upendo ni hisia kama vile huruma na wema kwa wengine. Pia ni mvuto unaohusisha watu wawili, mvuto wa kitu au jambo fulani. Upendo ni muhimu sana katika jamii kwani watu wanapopendana huishi kwa amani. Hii ni kwa sababu unapompenda mtu utaepuka kumtendea mabaya yatakayomuudhi na badala yake utamtendea yatakayomletea furaha. Kwa mujibu wa nadharia tunayoitumia ya Ajenda (McCombs \& Shaw), waandaaji wa kipindi cha kipindi cha Rauka cha runinga ya Citizen wanahimiza jambo hili la upendo kwa kila mmoja kwa kuteua nyimbo ambazo hubeba ujumbe huu wa upendo. Uteuzi huu wa nyimbo zinazowasilisha maudhui ya upendo na kupeperusha kwa watazamaji, huathiri maoni ya umma. Watazamaji wanapopata ujumbe huu kupitia vyombo vya habari hasa runinga, hawana budi kuufuata kwani huchukulia kuwa ni muhimu.

Mwimbaji Kambua katika wimbo wake Usiku na Mchana anaeleza kuwa Mungu ana upendo na umemgusa moyo wake. Anasema:

This love has captured my heart

This love has set me free

So I will shout about, I will dance about it

Nitatangaza upendo wako

Every tribe and every tongue

Raise your voice to him cry...

Upendo huu umegusa roho yangu

Upendo huu umeniweka huru

Nitatangaza kuihusu, nitacheza kwa furaha kuihusu 
Nitatangaza upendo wako

Kila kabila na kila ulimi

Inua sauti yako kwako umlilie...( T.Y)

Mwimbaji huyu anatuambia kuwa upendo huu wa Mungu umemgusa roho yake hivyo ataitangaza kwa sauti ya juu ili kila mja apate kuusikia. Nao waimbaji wa Zabron (Zabron singers) wanaeleza kuwa Mungu ni wa upendo. Wanasema hivi katika wimbo wao Mkono wa Bwana:

\section{Msalabani ulitufia \\ Hm, ulitupenda \\ Dhambi zetu ukabeba \\ Baraka zako tuliziona kweli tumeona \\ Hakika unabariki...}

Anasisitiza jambo hili la upendo kwamba Mungu si kama binadamu. Hana ubaguzi anapenda watu wote kwani wote ni sawa kwake. Mungu ni kielelezo kwa wanadamu hivyo ni muhimu kupenda. Tendo la kupendana litapunguza huzuni na uchungu kwa wahusika.

\section{Uvumilivu}

Uvumilivu ni uwezo wa kungojea mtu, kitu au jambo Fulani bila kukasirika na kwa matumaini kuwa utapata matokeo mazuri. Kwa mujibu wa Kamusi ya Kiingereza ya Collins (1979), uvumilivu ni uwezo wa kudhibiti hisia na mihemko na kuweza kuendelea kwa utulivu licha ya kukumbana na hali ngumu/matatizo. Uvumilivu ni sifa ya kimaadili ambayo hutusidia kutofanya maamuzi kwa haraka. Hutusaidia kukubali hali ya maisha hasa matatizo tunayokabiliana nayo katika maisha ya sasa tukitarajia kuwa siku za usoni zitakuwa bora. Mara nyingi mtu anapokumbwa na matatizo, watu wengi humshauri awe mvumilivu na mtulivu hata kabla ya kuwazia kuhusu suluhisho halisi la tatizo linalomkabili. Hii ni kwa sababu hali hii ya utulivu itamsaidia kuweza kufikiria vyema na kwa makini kuhusu suluhisho la tatizo linalomkabili badala ya kuwazia suluhisho kwa haraka ambalo litamletea madhara zaidi.

Ni muhimu kutaja kuwa uvumilivu ni maadili yanayothaminiwa sana katika dini nyingi kama vile ya Kikristo, Kiislamu, Kiyahudi, Kihindi na kadhalika. Katika dini ya Kikristo kwa mfano, uvumilivu ni mojawapo ya matunda ya roho mtakatifu (Wagalatia 5:22-23 Lakini tunda la Roho ni upendo, furaha, amani, uvumilivu, utu, wema, fadhili). Wakristo huamini kuwa Yesu ni kielelezo cha uvumilivu kwani alivumilia mateso mengi kabla ya kifo chake. Watu wasio na uvumilivu hukosa kuridhika, huwa na hali ya juu ya kupata maradhi ya moyo kutokana na kukasirika, hujiuzulu kazini kwa haraka, huvunja urafiki na wengine haraka na pia hukosa msukumo kwa haraka wa kufikia lengo lao hasa wanapopata changamoto. Matokeo haya yote yanaleta uchungu kwa anayehusika. Kwa upande mwingine, watu walio na uvumilivu hukosa kupata mfadhaiko kutokana na shida, hufurahia hatua ya kutimilika kwa jambo hata kama inachukua muda mrefu, huwa watulivu na wenye afya nzuri na hutimiza malengo yao kwa ufanisi wa hali ya juu. Haya yote ni matokeo yaletayo furaha.

Mwimbaji Rose Muhando anazungumzia maudhui ya uvumilivu katika wimbo wake Yesu karibu kwangu. Anahimiza kuvumilia ingawa anapata maumivu sana. Anasema:

\section{Kikombe nimekinywea \\ Hukumu nimechukua \\ Mateso nimeyapokea \\ Maumivu nimevumilia}

Anaposema kikombe nimekinywea anamaanisha kuwa shida ameyapata lakini alivumilia na kumwita Yesu akaribie maisha yake amsaidie.

Katika wimbo huu, mwimbaji anatumia muktadha wa kidini kuwaeleza watazamaji wa wimbo wake kuwa shida wazipatazo ni majaribio tu ambayo hatimaye yataisha. Katika dini, huaminika kuwa shida inapopatikana huwa ni majaribio ya shetani na kwa wakati kama huo; wanapaswa kuvumilia, kuomba na kuwa na imani kuwa Mungu atawawezesha kuyashinda yote.

Vigezo hivi vinadhihirisha kuwa tendo la kuvumilia linapunguza uchungu kwa mhusika. Kulingana na dini pia tendo la kuvumilia ni adilifu maanake dini tofauti zinahimiza watu kuwa wavumilivu. Mwimbaji kwa hivyo anahimiza jamii kuwa na uvumilivu kwani utawawezesha kukabiliana na matatizo mengi ambayo hukumba binadamu kila mara. 


\section{Shukrani}

Shukrani ni tendo la kutambua usaidizi au msaada ambao mtu amepata au anaotarajiwa kupata. Kwa mfano, kwa mujibu wa Carey na wenzake (1976), majaribio yaliyofanywa ya kuonyesha athari ya kutoa shukrani yalionyesha kuwa, wateja katika duka la mapambo ya vitu walioitwa na kisha kupewa shukrani yalionyesha kwa kununua walidhihirisha ongezeko la asilimia 70 la bidhaa walizonunua, wale ambao waliitwa tu lakini hawapewa shukrani kwa kununua walidhihirisha ongezeko la asilimia 30 la bidhaa walizonunua. Hatimaye, wale ambao hawakuitwa wala kupewa shukrani hawakudhihirisha ongezeko lolote la bidhaa walizonunua. Hili ni dhihirisho kuwa tendo la kushukuru lina manufaa kwa mtenda na mtendwa. Katika mfano huu, linasababisha furaha kwa mteja kiwango cha kumfanya anunue bidhaa zaidi. Hili linasababisha furaha kwa muuzaji maanake atauza bidhaa nyingi hivyo kupata faida kubwa.

Msanii Joel Lwaga anaangazia maudhui ya shukrani katika wimbo wake Umejua kunifurahisha. Katika stanza ya kwanza na ya pili, anasema Mungu amemfurahisha kwa kumfuta machozi ndiyo maana atamsifu milele. Hali hii ya kumsifu milele ni njia moja ya kutoa shukrani. Anaendelea kusema kuwa Mungu amemrudishia tabasamu lake usoni, kumrejeshea furaha moyoni na kumjibu maombi yake hivyo anamshukuru:

Umerudisha tabasamu langu

Umerejesha na furaha yangu moyoni

Umenijibu kwa wakati nisiyodhani

Baba umejua kunifurahisha...

Anasema kuwa Mungu alimpa baraka alipokuwa akiitwa laana, amemfanya aheshimike aliyeonekana hafai hivyo anamshukuru Mungu. Ubeti 4:

Niliyekuwa laana, nimefanyika Baraka

Niliyeonekana sifai, umeniheshimisha

Umenipa sababu ya kudai na kukutukuza aah

Baba umejua kunifurahisha

Hey baba!

Naye Kambua katika wimbo wake Usiku na mchana anatoa shukrani kwa Mungu. Yeye ndiye mwandaaji wa kipindi cha Rauka na alipeperusha wimbo huu kumshukuru Mungu kwa kumpa mtoto mvulana aliyekuwa amemtafuta kwa muda mrefu tangu aolewe. Aliongezea kwamba alimwamini Mungu kuwa atamtendea haki na kweli alisema alijibiwa maombi yake. Anasema hivi:

Usiku na mchana, nakupa sifa

Day and night Lord I'll give you praise

Kila niendapo, nitakuinua

I'll honor and live for you $\mathrm{x} 2$

I will praise you Lord you have made me glad

Lift your name up high let the whole world know

You have been my help my own time God Rafiki wa karibu

Nao waimbaji wa Zabron katika wimbo wao Mkono wa Bwana wanatoa shukrani kwa Mungu kutokana na matendo yake makuu. Wanasema:

Tumeuona mkono wako Bwana

Matendo yako Bwana ni makuu mno

Umetutoa mbali

Ukatushika mkono

Mahali uliona pekee hatuwezi

Chini hata juu

Watu unawainua

Sifa na utukufu ni zako milele

Twasifu jina lako

Milele tunakusifu

Daima we umwema tunaishi kwako

Mwandaaji wa kipindi anapitisha maudhui ya shukrani kwa watazamaji kupitia nyimbo za wasanii. Japo kuna nyimbo nyingi za wasanii wengi zinazozungumzia maudhui mbalimbali, wao waliteua mada na nyimbo ambazo tumetaja hapo juu. Kulingana na mhimili wa kwanza katika nadharia ya ajenda ya McCombs \& Shaw, vyombo vya habari huteua mada na kupeperushwa kwa watazamaji hivyo mada hiyo kuchukuliwa kuwa ni muhimu. Nyimbo zinazopeperushwa wakati wa kipindi huteuliwa na waandaaji wa kipindi na kupeperushwa hivyo watazamaji kuchukulia kuwa nyimbo hizo ni muhimu hata wakati mwingine wanalazimika kutafuta kanda za nyimbo hizo na kuzicheza mara kwa mara. Kupitia nyimbo hizi, jamii wanahimizwa kuwa wenye shukrani kwa Mungu na yeyote anakutendea mema katika maisha. 


\section{Heshima}

Katika utafiti wetu, neno heshima tumelichukulia kuwa ishara ya kuthamini. Ni hali ya kufanya adabu kwa Mungu au binadamu wengine. Wasanii Kambua na waimbaji wa Zabron wote wanasisitiza heshima kwa Mungu. Ujumbe huu ni wa muhimu sana kwani kwa kuzingatia heshima, kudumisha amani na kujiepusha migogoro mengineyo katika jamii. Maoni haya yanaongozwa na mhimili wa nadharia iliyotuongoza kuwa, "Vyombo vya habari hushughulikia masuala machache. Katika wimbo wa Kambua. Anasema haya:

Usiku na mchana nakupa sifa

Day and night Lord I'll give you praise

Kila niendapo nakuinua

I'll honor and live for you

Usiku na mchana nakupa sifa

Mchana na usiku Bwana nitakusifu

Kila niendapo nakuinua

Nitakuheshimu na kuishi kulingana na mapenzi yako.(T.Y)

Msanii huyu anaeleza umuhimu wa kuheshimu Mungu. Na waimbaji wa Zabron wanasema:

Sifa na utukufu zako milele

Twasifu jina lako

Milele tunakusifu

Daima we umwema tutaishi kwako

Waimbaji hawa wanasisitizia jamii kumheshimu Mungu ambaye anatambua kila jambo linalotokea na mwenye uwezo wa kuwapa waja wake mazuri wa kila aina. "Unatawala dunia na vyote vilivyomo, makuu umeyatenda..." Inasema kuwa Mungu ndiye anawasaidia wanadamu kwa kila jambo hivyo basi ni sharti aheshimiwe.

\section{Mateso}

Mateso ni kitu au jambo lolote linalokuumiza au kukutesa. Mateso yanaweza yakawa ya kiroho au kimwili. Katika Biblia tunapozungumzia mateso tunakumbuka watu kama Ayubu, Paulo na hata Yesu. Ayubu alipitia dhiki kubwa sana. Mali yake yote yalipotea, mifugo na watoto walikufa na akapatwa na ugonjwa mbaya sana ulioangamiza mwili wake. Baada ya mateso hayo Mungu alimponya na kumrejeshea afya, watoto wake na mali yote mara saba kwa sababu alimwamini. Paulo naye alikuwa mtu aliyewatesa Wafarisayo sana. Baada ya kubadilika na kuwa mtu anayemtii Mungu na kuwahubiria watu wale aliowatesa wakaanza kumtesa ili kulipiza kisasi kwa mabaya aliyowatendea mbeleni. Wayahudi walimtesa kwa sababu waliamini kuwa Paulo aliwafundisha watu mafunzo ambayo Wayahudi hawakufurahia wala kuamini. Mateso aliyoyapitia ni kama kupigwa, kuwekwa jela kwa miaka mitatu na nusu na mwishowe kuuawa kwa kukatwa shingo. Yesu kwa upande mwingine alipitia mateso kwa sababu alipokuwa akihubiri kuhusu wokovu, Wayahudi waliona kwamba anafundisha mambo yaliyo kinyume na imani yao. Vilevile, Yesu alikuwa na wafuasi wengi na vingozi waliokuwa wakiongoza wakati huo wakawa na wivu kwake hivyo wakamtesa kwa kukataliwa na ndugu na jamaa zake, alibebeshwa msalaba, alipigwa misumari mikononi mwake na kuvalishwa miiba kichwani mwake. Mateso ni hali yoyote ambayo inakunyima wewe kuchangamka. $\mathrm{Ni}$ kitu chochote kinachoweza kukukandamiza mwili ukasikia maumivu inaweza ikawa ajali, ugonjwa na kadhalika. Mateso pia ni ile hali inayokuzuia kwenda mbele aidha kusoma, unataka kufanya biashara pesa huna, inakunyima maendeleo yako. Zaburi 107:13 "Wakamlilia Bwana katika dhiki zao, Bwana akawaponya na shida zao zote." Mateso ni ile hali unayosikia ya kuugulia ndani ya moyo na huna njia ya kutatua hilo jambo. Binadamu ana matumaini kwamba baada ya dhiki ni faraja. Mateso yanaisha tukivumilia na kumwamini Mungu aliye na uwezo wa kuyaondoa mateso hayo. Hivyo waimbaji wengi wa nyimbo za dini wanapitisha ujumbe wa kuamini Mungu ili watusaidie kuyaondoa mateso.

Waandaaji wa kipindi cha Rauka wanapitisha ujumbe huu wa mateso kwa wanajamii kupitia nyimbo za wasanii mbalimbali kama vile Rose Muhando, Sifaeli Mwabuka na David Ekene. Rose Muhando anazungumza kuhusu mateso katika wimbo wake Yesu karibu kwangu. Anasema hivi:

Baba, baba...

Kikombe nimekinywea

Hukumu nimechukua

Mateso nimeyapokea

Maumivu nimevumilia 
Msanii huyu anaonekana ameonekana amepitia mateso mengi maishani ndipo anamkaribisha Yesu maishani mwake. Wakristo wengi wanaamini kwamba mtu anayapitia mateso kwa sababu ya dhambi alizozitenda hivyo kumwita Yesu maishani mwake ili aweze kusaidika kutokana na mateso aliyokuwa nayo. Naye Sifaeli Mwabuka katika wimbo wake Shuka tukuone Mungu wetu anaeleza kuhusu mateso. Anasema kwamba unapopitia changamoto katika maisha Mungu hushuka na kutatua shida yako. Anasema hivi:

Unaposema siwezi siwezi tena kusonga ndipo Mungu

Anashuka kukushika mkono wako. Unaposema siwezi

Siwezi tena kupona ndipo Mungu anashuka kukuponya

Magonjwa yako. Unaposema siwezi siwezi tena kushinda

Vita ndipo Mungu anashuka na majeshi ya mbinguni.

Unaposema siwezi siwezi tena kupata mwenza ndipo

Mungu anashuka kukuletea mwenza ndipo Mungu anashuka

Kukuletea mwenza wako. Unaposema siwezi siwezi tena

Kuinuliwa ndipo Mungu anashuka kutimiza ahadi zako.

Mateso aliyoyazungumzia msanii huyu ni kutoendelea kimaisha, magonjwa, kushindwa vita, kutopata mwenza na kutoinuliwa. Mwabuka aliendelea kuonyesha kuwa Mungu huwasaidia wanadamu waepukane na mateso. Anatoa mifano ya wana Israeli, Sara na Ezekieli. Anasema hivi:

Wana wa Israeli walipofika bara la Shamu akili zao zote zilifika mwisho.

Tukimkumbuka Sara hata alipofika uzeeni, Tumaini ya kupata mtoto lilifika mwisho $\mathrm{x} 2$.

Kuhani Ezekieli akili yake iliposhindwa

Mungu akashuka akamwita Ezekieli...

Kupitia wimbo huu, tunafahamu kuwa Mungu anawasaidia watu wake kutoka katika hali ngumu ya maisha. Wana wa Israeli walivuka bara la Shamu kwa usaidizi wake, akamwezesha Sara kupata mtoto katika uzeeni na pia akamsaidia Ezekieli. Naye David Ekene katika wimbo wake Greater Tomorrow anaelezea kuwa binadamu anapitia mateso mengi lakini Mungu atamwezesha kushinda. Anatueleza hivi:

His hands are not short

That He cannot reach to you

His eyes are never blind (that) He cannot see your tears

His ears are not deaf

That He cannot hear your cry

Your tomorrow must be greater than today...

Mikono yake si mafupi

Pasipo kufikia kwako

Macho yake si mapofu pasipoona machozi yako

Masiko yake si mazito

Siku yako ya kesho lazima iwe bora kuliko leo...(T.Y)

Kupitia maelezo haya yote tumefahamu kuwa kuna mateso katika jamii na wanajamii wanapohimizwa kupitia nyimbo zinazopeperushwa kwenye kipindi cha Rauka wanapata matumaini ya kuendelea na maisha japo kuna matatizo za kila siku.

\section{Msaada}

Msaada ni huduma au faraja anayopewa mtu anapokuwa na shida au tatizo kama vile ajali, matatizo ya kifamilia, msongo wa mawazo na kadhalika. Binadamu wote wanahitaji msaada katika maisha yao. Hivyo ni muhimu kujenga tabia ya kuwa tayari kuutoa na kuupokea vilevile. Mungu husaidia wakati wa taabu na dhiki hivyo ndivyo Sifaeli anatueleza katika wimbo wake, Shuka tukuone Mungu wetu . Anasema hivi:

Unaposema siwezi siwezi tena kusonga ndipo Mungu anashuka

Kushika mkono wako. Unaposema siwezi siwezi tena kupona

Ndipo Mungu anashuka kukuponya magonjwa yako.

Unaposema siwezi siwezi tena kushinda vita ndipo Mungu

Anashuka na majeshi ya mbinguni...

Katika wimbo huu, msanii anatueleza kuwa Mungu husaidia katika hali zote ngumu si magonjwa si vita na hivyo binadamu anahitajika kuamini kuwa atasaidika. Mwimbaji anasema kuwa jinsi alivyowasaidia wana wa Israeli katika bara la 
Shamu, Sara na Ezekieli anaweza kuwasaidia watu wote walio na shida. Mhimili wa nadharia ya ajenda ya McCombs na Shaw hueleza kuwa wahariri ndio wanateua habari ya kwenye vyombo vya habari. Inamaanisha kuwa kabla ya masuala yoyote kupeperushwa lazima wahariri wapitie. Hivyo, nyimbo ambazo zinapeperushwa kwenye zinateuliwa na wahariri kwa mwongozo wa waandaaji wa kipindi wakiwa na lengo la kupitisha ujumbe maalum kwa watazamaji wake. Ujumbe huu wa msaada uliwasilishwa kupitia nyimbo zilizochezwa kwenye kipindi cha Rauka kwa lengo la vyombo vya habari kuwahimiza watazamaji kwamba msaada unatoka kwa Mungu. Watazamaji watachukulia kuwa jambo hili ambalo limepitishwa kwenye kipindi ni muhimu na wataamini.

Naye David Ekene katika wimbo wake Greater Tomorrow anatueleza kuhusu msaada. Wimbo huu unaeleza imani aliyokuwa nayo msanii kwa Mungu. Anaamini kuwa Mungu ana uwezo wa kusaidia kila mja aliye na imani. Anasema hivi;

His hands are not short

That He cannot reach to you

His eyes are never blind(that)

He cannot see your tears

His ears are not deaf

That He cannot hear your cry...

Mikono yake si mifupi pasipo kufikia kwako, macho yake si

Mapofu pasipoona machozi yako, masikio yake si mazito

Pasiposikia kilio chako...(T.Y)

Msanii huyu anaeleza kuwa Mungu anaona shida anazopitia binadamu na anasikia maombi yake kisha anajibu hayo kwa kumsaidia mja wake kuepukana na shida zote zinazomkumba. Nao waimbaji wa Zabron katika wimbo wao Mkono wa Bwana wanatueleza kuwa Mungu ametenda yote mazuri kwa kuwa yeye ni muweza:

Mengi mazuri tumeyaona

Mungu umetenda

Ni kweli we muweza...

Wasanii hawa wote katika nyimbo zao wanaeleza kuwa Mungu anawasaidia watu hivyo hawafai kukata tamaa. Kwa hivyo wanajamii wanapata ujumbe huu kupitia nyimbo hizi zinazopeperushwa na waandaaji wa kipindi cha Rauka.

\section{Wema}

Wema ni hali ya kuwa na moyo wa upendo, huruma na kuwajali wengine au kuwathamini wengine. Ni hali ya kuwachukulia au kuwaona wengine kuwa ni bora na ni wa thamani kama unavyojichukulia. Ni kuepuka kuwatendea wengine kama jinsi unavyopenda kutendewa. Matendo mema huleta sifa njema kwa mtu kwani huwa yanakubalika katika kila jamii. Vyombo vya habari hushughulika katika kuteua ajenda kwa umma. Haya ni kwa mujibu wa McCombs na Shaw katika nadharia ya ajenda. Kupitia wimbo wa Rose Muhando na Olivia Wema, waandaaji wa kipindi cha Rauka walipitisha ujumbe huu wa wema katika wimbo wao Hayawe Hayawe. Ujumbe huu ni muhimu kwa watu wote katika jamii kwa sababu kila moja anapotenda mema kwa mwenzake ama jirani watakuwa na mlahaka mzuri wanapoishi pamoja. Wanasema hivi katika wimbo wao:

Wana heri wale wapelekao habari njema, $\mathrm{Na}$ midomo yao inatamka maneno mema...

\section{Kukata Tamaa}

Msanii Mwabuka anazungumzia kukata tamaa kwa wanadamu. Tamaa ni tendo la kukosa matumaini katika maisha. Anaeleza kuwa binadamu anapokosa matumaini Mungu anashuka kumaanisha anamsaidia kuepukana na taabu alizokuwa nazo. Anasema hivi:

...Unaposema siwezi siwezi tena kusonga ndipo Mungu anashuka kukushika

Mkono wako. Unaposema siwezi siwezi tena kupona ndipo Mungu anashuka. Kukuponya magonjwa yako. Unaposema siwezi siwezi tena kushinda vita. Ndipo Mungu anashuka na majeshi ya mbinguni. Unaposema siwezi siwezi. Tena kupata mwenza ndipo Mungu anashuka kukuletea mwenza wako.

Katika hii stanza, msanii anaeleza jinsi binadamu anakosa matumaini Mungu anashuka na usaidizi. Kwa mfano mtu mgonjwa anaposema hawezi tena kupona anaonyesha hali ya kukosa matumaini 
lakini Mungu anatoa msaada wa kuponya magonjwa yake.

\section{HITIMISHO}

Katika utafiti huu tumewasilisha, kujadili na kufasili data ya utafiti ili kutimiza malengo ya utafiti wetu. Tumebainisha maudhui kwenye nyimbo zinazopeperushwa kwenye kipindi cha Rauka kwa kuzingatia mihimili ya nadharia ya Ajenda. Kutokana na matokeo ya utafiti huu, tumehitimisha kuwa vyombo vya habari vina mchango katika uwasilishaji wa maudhui katika fasihi simulizi kupitia uhuishaji wa nyimbo. Nyimbo katika kipindi cha Rauka zinateuliwa kwa umakini mkubwa ili kuwasilisha ujumbe wenye uwezo wa kuimarisha maadili katika jamii.

\section{MAREJELEO}

Behr, R. L., \& Iyengar, S. (1985). Television news, real-world cues, and changes in the public agenda. Public Opinion Quarterly, 49(1), 3857.

Beja, S. K. (2000). Giriama funeral songs: a functional aesthetic study (Doctoral dissertation, University of Nairobi).

Carey, J. R., Clicque, S. H., Leighton, B. A., \& Milton, F. (1976). A test of positive reinforcement of customers. Journal of Marketing, 40(4), 98-100.

Collins, H. (1979). Collins English Dictionary. Great Britain: Harper Collins Publishers

MacFarland, D. (1990). Contemporary radio programming strategies. Hillsdale, $\mathrm{NJ}$ : Lawrence Erlbaom Associates.

Matteru, M. (1981). Fasihi simulizi na uandishi makala ya semina ya waandishi wa Kiswahili: Dar es salaam.

McCombs, M. E., \& Shaw, D. L. (1972). The agenda-setting function of mass media. Public opinion quarterly, 36(2), 176-187.

Senkoro, F. (1979). Fasihi; Press and Publicity Centre. Dar es salaam.
Wamitila, K. (2003). Kichocheo cha Fasihi: Simulizi na Andishi: Focus Publications Ltd.

Wamitila, K. (2008). Kanzi ya Fasihi Misingi ya Uchanganuzi wa Fasihi. Nairobi: Vide muwa Publishers Ltd.

Wanjala, F. (2013). Kitovu cha Fasihi Simulizi kwa Shule. Vyuo na Ndaki. Mwanza: Serengeti Educational Publishers(T) LTD.

Wanta, W. (1988). The effects of dominant photographs: An agenda-setting experiment. Journalism Quarterly, 65(1), 107-111.

Wasike, D. (2017). Lugha kama rasilimali ya jamii: Uchunguzi wa redio za FM zinazotumia kibukusu katika kaunti ya Bungoma. Tasnifu ya uzamifu chuo kikuu cha Moi.

Wasike, M. D. (2017). Lugha kama rasilimali ya jamii: uchunguzi wa redio za fm zinazotumia kibukusu katika kaunti ya bungoma (Doctoral dissertation, Moi University). 